\title{
DIGITAL PLATFORMS, AFFORDANCE, AND PUBLIC GOVERNABILITY
}

\author{
Leonid Smorgunov \\ St. Petersburg State University, 7/9 Universitetskaya emb., St. Petersburg, 199034, Russia
}

\begin{abstract}
Public governability on digital platform is an affordance effect arising in network structures with a wide participation of citizens. Network platforms provide collaboration based on an institutional form of interaction that is more effective than competitive representation. The digital institutional platform provides principles of equality, indifference, reciprocity and autonomy. A participatory public administration based on cooperation assumes an appropriate technical platform, which should have some qualities of affordance, i.e. the ability to act as a mediator for this type of interaction. The technological component, expressed in the idea of the state as a platform, is characterized by the presence of affordances for cooperation. The technology of blockchain is studied as the example. Blockchain is oriented towards procedural justice, which expresses the technological affordances of cooperation. Procedural justice is ensured by the affordability of technology that is created by blockchain algorithms. The paper compares the corresponding problems of affordance.
\end{abstract}

\section{KEYWORDS}

Digital Platforms, Public Governability, Collaboration, Affordance, Blockchain

\section{INTRODUCTION}

The use of network forms of coordination in economics and politics, based on digital technologies, has questioned the traditional forms of governability associated with centralization, representation, feedback and maintaining equilibrium with the environment. It is possible to believe that digitalization generates a new form of governability by increasing the number of governance participants organized into network platforms, and that a governability is a network effect based on the ability of the digital system to afford procedural fair norms. Affordance is an essential feature of technical systems that guide people to use them (Neff et al., 2012).

In the scientific literature, the objective function of participatory governability has been fixed. Firstly, participatory public governance is used not just as a form of democracy development, but is a response to the need for governability arising in the conditions of differentiation of interests and growing conflict in society (Kübler et al., 2019). Secondly, participatory public administration in the form of networks arises as the need to ensure governability in the context of increasing the role of innovations in the public sphere (Gil-Garcia et al., 2018). Thirdly, governance networks that attach greater importance to citizen participation tend to create an affordance policy, the concept of which is more comprehensive, and emphasize aspects of technical usability (Santos, Faure, 2018). Participatory governability is formed in certain technoecology and is expressed in reaching consensus based on a set of affordances. The main theoretical question of this article is that public governability in the context of digitalization, along with other factors, is determined by the system of affordances, which make digital platforms relevant to the network idea of their use. The article describes the system of affordances and considers their manifestation based on the study of the digital platform "Active Citizen" (Moscow).

\section{GOVERNMENT PLATFORM AND PARTICIPATORY GOVERNABILITY: TECHNOLOGY AND AFFORDANCE}

In the first decade of the existence of electronic governments in the world (conditionally 1995-2005) they were organized into Internet portals. The portals are less democratic, than Internet platforms based on 
interactive Web 2.0 technology and relational affordances. If bureaucratic administration uses technology, then they contribute to the affordances of information by passing the signal from top to bottom. Public management is incentive-oriented, and technology provides effective resource savings. Public governance, focused on interaction and co-governance, requires participatory affordances, ensuring coordination and reciprocity.

\subsection{Citizen Oriented Government Platforms}

Currently, the functioning of e-government is only partially organized in Internet portals, i.e. complex electronic means that provide for the implementation of a number of government functions and services for citizens. Internet portals provide search and information acquisition, interaction of citizens and organizations with state authorities, holding events for citizens and organizations, and providing public services. Internet platforms are complex electronic tools that provide not only services, but the participation of citizens in the choice of public services and influencing the decision-making process (O'Reilly, 2010). If we use the direct meaning of the concept of platform as an aggregate of ICT 2.0, oriented to the use of a web application system on one server for interactivity and personal participation, modern public governance includes such technologies under the flag of "ability for collaboration". In this respect, electronic platforms are an open and technical means of accordance for interrelations.

E-government now has characteristics that promote democracy in the broadest sense of the word, including civic participation virtually in the day-to-day activities of the state and in the processes of providing public services. In this respect, e-government becomes the basis for the network interaction of citizens, associations of civil society and business with the state in various areas of public activity. At this stage, the importance of assessing the electronic participation of citizens through the diverse sites of such e-government. As Kay Erickson and Henry Vogt rightly write, new digital forms of policy and management include network-based inputs for organizing all government information and public services in accordance with the needs and interests of certain segments of the population. This approach from the standpoint of "one window" is built on the concept of self-service with the requirement for users to become more active and self-governing (Eriksson, Vogt, 2013). There are three main areas for studying platform-based public administration. One of them puts emphasis on the formation of a special ecology of interaction between platforms and human agents in the public administration system (Van Dijck et al., 2018). Here affordances are the relational characteristics of the emerging technoecology. The second direction indicates the significant role of algorithms and artificial intelligence in platform control processes (Gillespie, 2010). Considering algorithms as a specific system of norms, researchers pay attention to the coordinating function of algorithmic control. Accordingly, affordances are considered by them as properties of the structure and architecture of algorithmic control. The third area of research includes the role of companies producing digital public administration technologies in the scope of digital public administration (Gorwa, 2019).

Although the theme of affordances is subordinate to the political role of information companies, here too, the possibility of a democratic or authoritarian structural filling of algorithms and interfaces takes on its significance. Describing platforms and affordances, we will follow some integrated research strategy, emphasizing that the affordances of participatory governability are determined by all three conditions of digital public administration - technoecology, algorithms, and digitalization policies.

Platforms are technical and information conditions for increasing the self-organization of communities formed in networks. However, their quality is determined by certain conditions related to the orientation of the algorithms for the admission of interaction in the form of collaboration, i.e. the affordances of technology as a mediator, not as an intermediary. The difference between intermediaries and mediators, made by Bruno Latour with respect to mediating things [Latour, 2005], is that intermediaries are simple transfer structures between action agents, while mediators have their own agent binding policies, introducing a transformation into this connection. In this regard, platforms are mediators, manifesting their agent qualities through their performative functions. To perform these functions, they must possess some relational qualities that create a sociotechnical interaction ecology. To select the performance functions of digital technologies for public administration, we used the data obtained on the basis of the study of works that describe platform functions [Janowskia, 2019]. Analysis of different types of affordances allows you to correlate these functions, affordances and their location. The latter is conceptualized on the basis of two types of understanding of affordances: (1) as the qualities of technology; (2) as relational characteristics inherent in technoecology. A mixed source suggests that affordances are the result of both the qualities of technology and its involvement in the interaction of various socio-technical assemblies. 
Table 1. The affordances of citizen oriented government platform

\begin{tabular}{lll}
\hline Performative functions & Affordances & Location \\
\hline administer & normalize ability & algorithm \\
steer & programmability & algorithm \\
regulate & standardization & algorithm \\
serve & interoperability & mixed \\
engage & availability & technoecology \\
transform & ability to choose & technoecology \\
legitimize & identifiability & mixed \\
disclose & multimediality & mixed \\
monitor & persistence & mixed \\
participate & association ability & technoecology \\
empower & voice ability & mixed \\
learn & ability to share knowledge & mixed \\
coordinate & ability to stair a link & algorithm \\
create & ability to perceive new & mixed \\
collaborate & reciprocity & technoecology \\
& &
\end{tabular}

In the table. 1 you can see the results of a conceptual analysis of the relationship between the performative functions of digital platforms, affordances and the space of their appearance. It is clear that the functions of administering, general management (steering) and regulation with the corresponding affordances appear as a result of the introduction of the necessary algorithms into digital public governance. Whereas the functions of engagement, transformation, participation and collaboration involve the formation of such assemblages of human and technological agents that give rise to the corresponding technoecology as a space for the formation of relational affordances. Other functions and affordances suggest a mixed co-way of appearance and perception.

\subsection{Institutions of Affordance for Participatory Governability}

Digitization of public administration is a process of transformation of culture, organization and relations of public authorities with business and society through the use of new digital technologies (big data, internet things, artificial intelligence). Understanding the digitalization process as a way to transform the public governance space is determined not only by an orientation towards greater sensitivity and accountability, but also by a citizen-oriented government organization.

Digitization of public administration is often understood as the process of using new ICTs and, in particular, electronic technologies to organize and ensure the effective functioning of the public sphere. Moreover, it is emphasized that digitalization in the political sense is a way to expand the political forms of interaction between society and the state, making public governance collaborative. At the same time, it is important to note that citizen participation contributes to the development of "liquid democracy" in local communities - when political actors choose and vote on important issues - and this indicates that this issue is beginning to be on the political agenda. This type of democracy allows not only direct participation in decision-making processes, but also creates the conditions under which the representation of interests takes on the character of "tied reputation" secured by participation in the co-production of political decisions.

The value of digitalization goes far beyond simple control engineering. The regulatory framework used by digital technology allows us to solve a number of problems arising from the crisis of representative democracy, accountability and control. Analysis of the blockchain technology shows that it creates the affordances for a pure procedural justice, providing the possibility of an honest solution of public issues (Smorgunov, 2018). This example demonstrates the enormous possibilities of digitization of governance processes for the formation of its new organization. The previous choice was based on minimizing the costs of interdependence (external costs and transaction costs), while public governability was based on the affordances of the system to ensure security and be sensitive to basic interests. Digitalization does not diminish these requirements, but goes further, providing governability as mediator through a technological algorithm and collaborative technoecology. Governability as a network effect of digitalization of public administration is based both on the number of possibilities to interact and on the inclination to just public communication. It is supported by a procedure of fair consensus based on the affordance capacities of blockchain algorithm for the institutions of reciprocity, equality, indifference and autonomous organization. 


\section{BLOCKCHAIN AFFORDANCES AND COLLABORATIVE GOVERNABILITY}

Distributed data technology is gaining popularity in the economy and public policy. In the latter, it is used in the processes of voting, making decisions, determining agenda, policy evaluating, and other areas. The general belief of researchers is that the blockchain is not just a technology that increases the effects of economic production and political interaction, but is an institution that creates new affordances for the coordination of interactions.

\subsection{Blockchain Protocols and Rules of Justice}

As an example, confirming the presence in the protocols of digital technology norm, providing a fair consensus, we give the blockchain protocols. Currently, the important conditions for the technical support of fairness of interactions and decisions in the networks of the distributed register are the various algorithms included in the protocols and ensure the normal operation of mining. We turn here to some of them. Mining is the procedure for selecting a leader who has solved the task of agreeing the entries to the network in the form of another block that is recognized as valid for continuing the blockchain. Primary research on ontological questions of blockchain (philosophical, political, sociological) formed the idea of a transition from an institutional governance architecture with representation and hierarchy (centralism), to a procedural one, based on a humanitarian and technical platform of networked cooperation with free identification organized by anarchy, and distributed knowledge. In this respect, the question was raised whether the blockchain system is a complex system with nonlinear constraints that create affordances for the algorithmization of control and operation within it, or if we can speak about a complex but algorithmic blockchain-governance.

For this, certain validation procedures are formed. In the Bitcoin system, such a confirmation procedure was the Proof of Work, which was based on the principle of choosing a leader by the criterion of the speed of solving the task of forming the next block. For the formation of the next block leader (miner) received a reward in the form of cryptocurrency. Since the formation task was voluminous, it required the use of large computer capacities and, consequently, a lot of electricity. This system has a number of problems of economic, environmental and procedural properties. One of the procedural problems is related to the possibility of forming a monopoly (51\% of the combined miners) within a distributed network, which makes decisions in its favor. Although this is unlikely due to the possibility of confronting a monopoly on the output of honest users, it is not impossible. Therefore, other proof systems arose on other grounds. Proof on the basis of the rate (Proof of Stake) is constructed in proportion to the rate that everyone owns in accordance with the current blockchain register (Ethereum, Cardano). The main problem on the basis of PoS is the ability to imitate the process of choosing leaders in favor of one side. According to the developers of the improved Ourobokos blockchain proof protocol of the Cardano type, their addition to the protocol contributes to the viability and persistence of honest transactions (Kiayias, 2017). Often, this system is supplemented by methods for ensuring resiliency based on solving the Byzantine problem (EOSIO, BOScoin): security is achieved if two thirds of validators in the system are honest.

The question arises: what does the blockchain technology provide for relationships between people? Partnership or collaboration? Of course, at first glance, partnership is a collaboration. Often these terms are used interchangeably. Meanwhile, the practice of partnership and collaboration allows us to divide the content of these forms of interaction and find their distinctive features. Differences between these terms should be considered when comparing contexts in which these relationships occur. Somewhere the main dividing line is the difference between "being together" (being partners) and "acting together" (collaborating) (Carnwell, Carson, 2008). For a clearer separation of these concepts, we can probably say that a partnership is a joint activity based on distributed (often equal) rights and duties aimed at achieving common goals, whereas collaboration is a joint activity that proceeds from the unconditional desire of the interacting parties to work together to achieve common interests. The partnership is loaded with external legal conditions for joint activities, and collaboration implies an internal willingness to act together, subject to mutual assistance and responsibility. 


\subsection{Affordances of "Active Citizen" Platform}

One of the important areas of use of the blockchain is the "Active Citizen" platform, which was initiated in Moscow (Russia) to involve citizens in the choice of prioritized areas for the development of urban life in the capital, and to address topical current issues. This platform is part of the system of interaction between the city authorities and citizens, called system of joint decisions "Together". The joint decision system, formed by the mayor of Moscow for citizens' participation in the affairs of the city, includes a number of platforms related to the open government, open data, government services, as well as platforms for organizing the direct participation of citizens in public policy.

The "Active Citizen" is a platform for conducting open referendums in electronic form, created on the initiative of the Moscow Government in 2014. The project allows people to conduct citywide and local voting on a wide range of topics. Every week, Muscovites are invited to discuss important issues for the city. On the platform, a habitant needs to register, and to participate in a local vote, and must specify his address. For the passage of each vote, the participant is awarded points. Having gained 1000 points, he attains the status of "Active Citizen" and the opportunity to exchange them for city services (parking hours, visits to theaters and museums) or useful souvenirs. Bonus points can be earned also if he visits the application more often, invites friends, and shares information about the passed votes in social networks.

To solve the task of ensuring the transparency of the project, a number of tools have been implemented that allow users to monitor the progress of voting and monitor the reliability of the results obtained. In particular, each user who participated in the voting can: check the correctness of recording their vote; in online mode, monitor the overall dynamics of voting results. For these purposes, anyone can install a special program based on the system of the Ethereum, that is, everyone can become a member of the network of blockchain. This program allows network participants to see in real time those questions which have passed, or are still being voted on, as well as the appearance of new votes. The system allows anyone to become a member of the blockchain network not only as a resident of Moscow, but also as an organization. Blockchain, as indicated by the organizers of the program, affords for a number of additional performative functions: check the chronology of the appearance of votes and confirm their uniqueness; control the distribution of votes on issues; see the voices of real people (personal data is encrypted).

In 2018, this system in Moscow offered the use of multi-apartment housing blocks for citizens to vote. For this purpose, the "Electronic House" project is being implemented. This project is based on the affordance of Ethereum to form the decentralized autonomous organizations (DAO). In these last two cases of using blockchain technology to monitor the progress of voting and to make decisions, a number of characteristics of distributed network affordances that are related to the problem of procedural justice are seen. First of all, this is the honesty of the conditions of the emerging consensus, determined by the technological characteristics of distributed networks, and a small degree of identification in connection with the cryptographic protocols of recording and presenting users. Secondly, it is the acceptance of the terms of interaction that ensure the implementation of reciprocity affordance for the exchange of goods, decisions, knowledge and supervision. In this respect, the blockchain is a platform of collaboration for reciprocal benefits, whether it concerns finances, things, norms or regulation. Fair interaction affordances, together with a reciprocity, create a form of procedural justice in which incentives for genuine collaboration arise.

\section{CONCLUSION}

Recently, the concept of "co-creation" and "co-production" has been actively used in political science, public administration, and sociology as an innovation. Initially, this concept established a new attitude to the provision of public services and was directed against the market approach to the organization of this activity (Gil-Garcia, 2018). New breath she acquired in the development of the movement for digital governance. The concept of "co-production" is common in the study of public policy in general. Co-production is a new position for democratic public policy in conditions of uncertainty and complexity.

Some researchers focused on presenting research materials related to "governing through collaboration", "co-creation" and "co-production" of public policy not only on the above aspects of the topic, but also on the impact of collaboration in public policy that would correspond to sustainable and inclusive development (Krenjova, Raudla, 2017). In this respect, a distinction is made between co-production based on technological 
intermediaries and co-production based on a technological affordance for partnership and collaboration. The latter takes the form of participation on the basis of identification with the objectives of public programs and responsibility for their implementation (O'Brien et al., 2017).

New digital public administration platforms ensure co-production in public policy with such basic norms that make citizen participation open, fair and reasonable. On this basis, participatory governability will mean joint action to develop society, when the network effect of collaboration generates the value of participation. In this case, the participatory governance is the higher, the more technology affords communication. Of course, there are many challenges and threats in this way of introducing digital affordances into the public sphere. However, they are rather temporary inconsistencies in the process of formation of modern culture, organization and forms of interaction provided by digitalization.

\section{ACKNOWLEDGEMENT}

This work was supported with a grant from the Russian Science Foundation (grant 19-18-00210 "Political ontology of digitalization: Study of institutional bases for digital forms of governability").

\section{REFERENCES}

Carnwell, R. and Carson A., 2008. The Concepts of Partnership and Collaboration. Effective Practice in Health, Social Care and Criminal Justice: A Partnership Approach. 2nd ed. Ed. by R. Carnwell, J. Buchanan. Open Universities Press, Maidenhead, USA, pp. 3-21.

Eriksson, K. and Vogt, H., 2013. On self-service democracy: Configurations of individualizing governance and self-directed citizenship. European Journal of Social Theory, Vol. 16, No 2, pp. 153-173.

Gil-Garcia, R. et al., 2018. Digital government and public management research: finding the crossroads. Public Management Review, Vol. 20, No 5, pp. 633-646.

Gillespie, T. 2010. The politics of "platforms". New Media \& Society, Vol. 12, No 3, pp. 347-364.

Gorwa, R., 2019. What is platform governance? Information, Communication \& Society, Vol. 22, No 6, pp. 854-871.

Janowskia, T. et al, 2019. Platform governance for sustainable development: Reshaping citizen administration relationships in the digital age. Government Information Quarterly [online], 1-49.

Kiayias, A. et al, 2017. Ouroboros: A Provably Secure Proof-of-Stake Blockchain Protocol. Criptography ePrint Archive, pp. 1-52.

Krenjova, J. and Raudla, R., 2017. Policy Diffusion at the Local Level: Participatory Budgeting in Estonia. Urban Affairs Review, Vol. 3, No 4, pp. 1-29.

Kübler, D. et al, 2019. Strengthen governability rather than deepen democracy: why local governments introduce participatory governance. International Review of Administrative Sciences, Vol. 0, No 0, 2019, pp. 1-18.

Neff, G. et al, 2012. Affordances, Technical Agency, and the Politics of Technologies of Cultural Production. Journal of Broadcasting \& Electronic Media, Vol. 56, No 2, pp. 299-313.

O'Brien, D. et al, 2017. Uncharted Territoriality in Coproduction: The Motivations for 311 Reporting. Journal of Public Administration Research and Theory, Vol. 27, No 2, pp. 320-335.

O'Reilly, T., 2010. Government as a Platform. Open Government: Collaboration, Transparency, and Participation in Practice. O'Reilly Media, Sebastopol, Calif., USA, pp. 11-40.

Santos, M. and Faure, A., 2018. Affordance is Power: Contradictions Between Communicational and Technical Dimensions of WhatsApp's End-to-End Encryption. Social Media \& Society, July-September, [online], pp. 1-16.

Smorgunov, L., 2018. Blockchain and a Problem of Procedural Justice of Public Choice. Digital Transformation and Global Society. Third International Conference, DTGS 2018, St. Petersburg, Russia, May 30 - June 2, 2018, Revised Selected Papers, Part I. Springer International Publishing, Ham, Swizerland, p. 13-23.

Van Dijck, J. et al, 2018. The Platform Society: Public Values in a Connective World. Oxford University Press, New York, USA. 